\title{
Refining the Galerkin method error estimation for parabolic type problem with a boundary condition
}

\author{
Alisher Mamatov ${ }^{1 *}$, Xusanboy Narjigitov ${ }^{2}$, Dilshod Turdibayev ${ }^{2}$, and Jamshidbek \\ Rakhmanov $^{2}$ \\ ${ }^{1}$ Tashkent Institute of Textile and Light Industry, 100100 Tashkent, Uzbekistan \\ ${ }^{2}$ Department of Mathematics, Gulistan State University, Gulistan, Uzbekistan
}

Abstract. The article considers a parabolic-type boundary value problem with a divergent principal part, when the boundary condition contains the time derivative of the required function:

$$
\left\{\begin{array}{c}
u_{t}-\frac{d}{d x_{i}} a_{i}(x, t, u, \nabla u)+a(x, t, u, \nabla u)=0, \\
a_{0} u_{t}+a_{i}(x, t, u, \nabla u) \cos \left(v, x_{i}\right)=g(x, t, u), \quad(x, t) \in S_{t}, \\
u(x, 0)=u_{0}(x), \quad x \in \Omega
\end{array}\right.
$$

Such nonclassical problems with boundary conditions containing the time derivative of the desired function arise in the study of a number of applied problems, for example, when the surface of a body, whose temperature is the same at all its points, is washed off by a well-mixed liquid, or when a homogeneous isotropic body is placed in the inductor of an induction furnace and an electro-magnetic wave falls on its surface. Such problems have been little studied, therefore, the study of problems of parabolic type, when the boundary condition contains the time derivative of the desired function, is relevant. In this paper, the definition of a generalized solution of the considered problem in the space $\widetilde{\mathrm{H}^{1,1}}\left(Q_{T}\right)$ is given. This problem is solved by the approximate BubnovGalerkin method. The coordinate system is chosen from the space $H^{1}(\Omega)$. To determine the coefficients of the approximate solution, the parabolic problem is reduced to a system of ordinary differential equations. The aim of the study is to obtain conditions under which the estimate of the error of the approximate solution in the norm $H^{1}(\Omega)$ has order $O\left(h^{k-1}\right)$. The paper first explores the auxiliary elliptic problem. When the condition of the ellipticity of the problem is satisfied, inequalities are proposed for the difference of the generalized solution of the considered parabolic problem with a divergent principal part, when the boundary condition contains the time derivative of the desired function and the solution of the auxiliary elliptic problem. Using these estimates, as well as under additional conditions for the coefficients and the function included in the problem under consideration, estimates of the error of the approximate solution of the Bubnov-Galerkin method in the norm $H^{1}(\Omega)$ of order $O\left(h^{k-1}\right)$ for the considered nonclassical parabolic problem with divergent principal part, when the boundary condition contains the time derivative of the desired function.

*Corresponding author: maz54@mail.ru 


\section{Introduction}

When studying a number of topical technical problems, it becomes necessary to study mixed problems of parabolic type, when the boundary condition contains the time derivative of the desired function. Problems of this type occur, for example, when a homogeneous isotropic body is placed in the inductor of an induction furnace and an electromagnetic wave is incident on its surface. Some nonlinear problems parabolic with the boundary condition containing the time derivative of the desired function has been viewed, for example, in papers [1-3]. Many scientists were engaged in the construction of an approximate solution by the Galerkin method and obtaining a priori estimates for an approximate solution for parabolic classical quasilinear problems without a time derivative in the boundary condition: Mikhlin S.G., Douglas J. Jr, Dupont T., Dench JE, Jr, Jutchell L and other [4-9]. And quasilinear problems, when the boundary condition contains the time derivative of the required function using the Galerkin method, were studied in [10-13].

\section{Methods}

In this paper, we consider a quasilinear problem of parabolic type, when the boundary condition contains the time derivative of the required function:

$$
\left\{\begin{array}{c}
u_{t}-\frac{d}{d x_{i}} a_{i}(x, t, u, \nabla u)+a(x, t, u, \nabla u)=0 \\
a_{0} u_{t}+a_{i}(x, t, u, \nabla u) \cos \left(v, x_{i}\right)=g(x, t, u), \quad(x, t) \in S_{t}, \\
u(x, 0)=u_{0}(x), x \in \Omega
\end{array}\right.
$$

where $\Omega$-bounded domain in $\mathrm{E}_{m}, m=\operatorname{dim}-$ the dimension of the domain $\Omega$,

$$
\begin{aligned}
& Q_{T}=\{\Omega \times[0, T]\}, \quad S_{T}=\{\partial \Omega \times[0, T]\}, \quad a_{0}=\text { const } \\
& >0
\end{aligned}
$$

A generalized solution from the space $\widetilde{\mathrm{H}^{1,1}}\left(Q_{T}\right)=\left\{u \in H^{1,1}\left(Q_{T}\right): a_{0} u_{t} \in L_{2}\left(S_{T}\right)\right\}$ to problem (1) is a function from $\widetilde{\mathrm{H}^{1,1}}\left(Q_{T}\right)$ satisfying the identity

$$
\begin{gathered}
\int_{Q_{T}}\left(u_{t} \eta+a_{i}(x, t, u, \nabla u) \eta_{x_{i}}+a(x, t, u, \nabla u) \eta\right) d x d t+ \\
+\int_{S_{T}}\left(a_{0} u_{t}+g(x, t, u)\right) \eta d x d t=0 \\
\forall \eta \in H^{1,1}\left(Q_{T}\right)
\end{gathered}
$$

We construct an approximate solution of Galerkin [14-16]. We take a coordinate system from the space $H^{1}(\Omega)$. We will seek an approximate solution $\mathrm{U}(\mathrm{x}, \mathrm{t})$ in the form $U(x, t)=\sum_{k=1}^{n} C_{k}^{n}(t) \varphi_{k}(x)$

where $\mathrm{C}_{k}^{n}(t)$ are determined from the system of ordinary differential equations

$$
\begin{gathered}
\left(U_{t}, \varphi_{j}\right)_{\hat{L}_{2}}+\left(a_{i}(x, t, U, \nabla U), \varphi_{j x_{i}}\right)_{\Omega}+\left(a(x, t, U, \nabla U), \varphi_{j}\right)_{\Omega}= \\
=\left(g(x, t, U), \varphi_{j}\right)_{S}, \quad j=\overline{1, n}
\end{gathered}
$$

with initial conditions

$$
\left(U(x, O)-u_{0}, \varphi_{j}\right)_{H^{1}(\Omega)}=0
$$

Here $\hat{L}_{2}(\Omega)$ - is the space of a function with scalar product $(u, v)_{\hat{L}_{2}}=(u, v)_{\Omega}+a_{0}(u, v)_{s}$ 
The purpose of this article is to find out the conditions under which the estimate of the error of the approximate solution in the norm $H^{1}(\Omega)$ has order $O\left(h^{k-1}\right), 1 \leq k \leq r$ we assume that the set $M=M_{h}$ is chosen from the family $S_{h}^{r}$.

\section{Results}

We first investigate the following elliptic problem: Find a function $W(x, t) \in M$ satisfying the integral identity [17-18]

where $\mathrm{u}$ is the solution to problem (2).

$$
\begin{aligned}
& \left(a_{i}(x, t, u, \nabla u)-a_{i}(x, t, u, \nabla W), v_{x_{i}}\right)_{\Omega}+\lambda(u-W, v)_{\Omega}= \\
& (g(x, t, u)--g(x, t, W), v)_{s}, \quad \forall v \in M
\end{aligned}
$$

Suppose that $\lambda$ is such a sufficiently large positive number that problem (3) has a unique solution.

In what follows, assume that the following norms are bounded [19-21] $\|u\|_{\left(L_{\infty}\left(o, T ; L_{\infty}(\bar{\Omega})\right)\right.}, \quad\|\nabla u\|_{\left(L_{\infty}\left(o, T ; L_{\infty}(\Omega)\right)\right.}, \quad\|W\|_{\left(L_{\infty}\left(o, T ; L_{\infty}(\bar{\Omega})\right)\right.}, \leq$

$\|\nabla W\|_{\left(L_{\infty}\left(o, T ; L_{\infty}(\bar{\Omega})\right)\right.} \leq \mathrm{K}=$ const,

Let $u \in H^{k}(\Omega), 1 \leq k \leq r$, and $W(x, t)$ be solutions to problem (2) and (3), respectively. The set $M=M_{h}$ selected from the family $S_{h}^{r}$. In addition, let the condition be satisfied:

$$
\left(p_{i}-q_{i}\right)\left(a_{i}(x, t, u, \mathrm{p})-a_{i}(x, t, u, \mathrm{q})\right) \geq c(p-q)^{2}, \mathrm{c}=\mathrm{const}>0
$$

and the functions $a_{i}(x, t, u, \nabla u)$ satisfy the Lipschitz condition in $\nabla u$, and the functions $g(x, t, u)$ - in $u$. Then the inequality is true

where $\eta=u-W$

$$
\|\eta\|_{H^{\prime}(\Omega)} \leq C\|u\|_{H^{k}(\Omega)} h^{k-1}, \quad 1 \leq k \leq r
$$

In addition, let the solution of problem (2) satisfy

$u(x, t) \in L_{\bar{q}}\left(O, T, H^{k}(\Omega)\right)$,
$\quad u_{t} \in L_{2}\left(O, T, H^{k}(\Omega)\right) \cap L_{\bar{p}}\left(O, T, L_{\tilde{p}}(\Omega)\right) \cap L_{\bar{p}}\left(O, T, W_{q}^{1}(\Omega)\right), \quad 1 \leq k \leq r$

where

$$
\begin{gathered}
\bar{p}=\frac{2 \bar{q}}{\bar{q}-2 \gamma}, \quad \bar{q} \geq 2 ; \quad q=2+\frac{2 \gamma}{1-\gamma} ; \\
0<\gamma \leq \alpha \leq 1, \quad \tilde{p}=\frac{2 \tilde{q}}{\tilde{q}-2 \gamma}, \\
\tilde{q} \in\left\{\begin{array}{cc}
{\left[2, \frac{2(m-1)}{m-2}\right],} & m \geq 3 \\
{[2, \infty)} & m=2
\end{array}\right.
\end{gathered}
$$

And let conditions (4) and the ellipticity condition be satisfied

$$
v \sum_{i=1}^{m} p_{i}^{2} \leq \frac{\partial a_{i}}{\partial p_{j}} p_{i} p_{j} \leq \mu \sum_{i=1}^{n} p_{i}^{2}, \quad \forall p \in E_{m}
$$

In addition, we assume that the functions $\frac{\partial a_{i}}{\partial t}, \frac{\partial a_{i}}{\partial u}, \frac{\partial a_{i}}{\partial p}$ satisfy the Hölder condition with respect to $\nabla \mathrm{u}$ with exponent $\alpha$, and the functions $\frac{\partial g}{\partial t}, \frac{\partial g}{\partial u}$ - in $\mathrm{u}$.

If the set $M=M_{h}$ belongs to the family $S_{h}^{r}$, then the estimate occur

$$
\left\|\frac{\partial \eta}{\partial t}\right\|_{L_{2}\left(o, T, H^{1}(\Omega)\right)} \leq C h^{\gamma(k-1)}
$$




$$
\begin{gathered}
\text { where } C=C(R)\left(1+\left\|\frac{\partial u}{\partial t}\right\|_{L \bar{p}(o, T, L \tilde{p}(\mathrm{~S}))}+\left\|\frac{\partial u}{\partial t}\right\|_{L_{\bar{p}}\left(o, T, W_{q}^{1}(\Omega)\right)}\right) \times\left(\|u\|_{L \bar{q}\left(o, T, H^{k}(\Omega)\right)}+\right. \\
\left.\left\|\frac{\partial u}{\partial t}\right\|_{L_{2}\left(o, T, H^{k}(\Omega)\right)}\right)
\end{gathered}
$$

Theorem. Let $u, \cup, W$ be solutions to problems (2), (3) and (4), respectively. Suppose that conditions (5) - (10) are satisfied. Suppose also that on the set

$$
\left\{(x, t) \in \bar{Q}_{T},\|v\|_{L_{\infty}\left(0, T, L_{\infty}(\bar{\Omega})\right)},\|\nabla v\|_{L_{\infty}\left(0, T, L_{\infty}(\Omega)\right)} \leq R\right\}
$$

the functions $\frac{\partial a_{i}}{\partial t}(x, t, v, \nabla v)$ are bounded, the function $a(x, t, v, \nabla v)$ satisfies the Lipschitz condition with respect to $v$ with respect to $\nabla v$, and $g(x, t, v)$ - with respect to $v$, moreover, let $U(x, o)$ satisfy the inequality

Then, the following estimate is true

$$
\|u(x, 0)-U(x, 0)\|_{\widetilde{L_{2}}} \leq C h^{(k-1)},
$$

$$
\|u-U\|_{L_{\infty}\left(O, T, \widetilde{L_{2}}\right)}+\|u-U\|_{L_{2}\left(O, T, H^{1}(\Omega)\right)} \leq C h^{\gamma(k-1)}, 1 \leq k \leq r
$$

relation (12) is true for $\mathrm{C}$.

Proof. Let us integrate equation (3) from 0 to t. Subtract the resulting equation from equation (2). Suppose that $\zeta=\eta+\xi$ and taking into account equation (4), we arrive at the identity

$$
\begin{aligned}
\int_{0}^{t}\left(\xi_{t}, v\right)_{\widetilde{L_{2}}} d t+\int_{0}^{t} & \left(a_{i}(x, t, U, \nabla W) d t+a_{i}(x, t, U, \nabla U), v_{x_{i}}\right) d t \\
& =\int_{0}^{t}\left(a_{i}(x, t, U, \nabla W)+a_{i}(x, t, u, \nabla W), v_{x_{i}}\right)_{\Omega} d t \\
& -\int_{0}^{t}\left\{\left(\frac{\partial \eta}{\partial t}, v\right)_{\widetilde{L_{2}}}+(a(x, t, u, \nabla u)-a(x, t, U, \nabla U), v)_{\Omega}+\lambda(\eta, v)_{\Omega}\right. \\
& \left.-(g(x, t, W)-g(x, t, U), v) \quad{ }_{s}\right\} d t, \quad \forall v \in M
\end{aligned}
$$

For a test function $v$ we take $v=\xi(\cdot, t)$ The left-hand side of equality 14) is estimated from below using assumption (5)

$$
\int_{0}^{t}\left(a_{i}(x, t, U, \nabla W)-a_{i}(x, t, U, \nabla U), \xi_{x_{i}}\right)_{\Omega} d t \geq \gamma_{1}\|\nabla \xi\|_{L_{2}\left(o, t, L_{2}(\Omega)\right.}^{2}
$$

We estimate the terms on the right-hand side of (14) from above using the Cauchy inequalities and the assumptions of the theorem as follows

$$
\begin{gathered}
\int_{0}^{t}\left|\left(a_{i}(x, t, \cup, \nabla W)-a_{i}(x, t, u, \nabla W), \xi_{x_{i}}\right)_{\Omega}\right| d t \leq \\
\leq \varepsilon\|\nabla \xi\|_{L_{2}\left(0, t, L_{2}(\Omega)\right)}^{2}+C \int_{0}^{t} \int_{\Omega}\left[a_{i}(x, t, \cup, \nabla W)-a_{i}(x, t, u, \nabla W)\right]^{2} d x d t \leq \\
\leq \varepsilon\|\nabla \xi\|_{L_{2}\left(0, t, L_{2}(\Omega)\right)}^{2}+C\left(\|\eta\|_{L_{2}\left(0, t, L_{2}(\Omega)\right)}^{2}+\|\xi\|_{L_{2}\left(0, t, L_{2}(\Omega)\right)}^{2}\right) \\
\int_{0}^{t}\left|(a(x, t, u, \nabla u)-a(x, t, \cup, \nabla \cup), \xi)_{\Omega}\right| d t \leq C\left(\|\xi\|_{L_{2}\left(0, t, L_{2}(\Omega)\right)}^{2}+\|\eta\|_{L_{2}\left(0, t, H^{1}(\Omega)\right)}^{2}\right)+ \\
+\varepsilon\|\nabla \xi\|_{L_{2}\left(0, t, L_{2}(\Omega)\right)}^{2}
\end{gathered}
$$


Similarly, we have

$$
\begin{gathered}
\left|\int_{0}^{t} \int_{s}(g(x, t, W)-g(x, t, \mathrm{U})) \xi d x d t\right| \leq C \int_{0}^{t} \int_{s} \xi^{2} d x d t \leq \varepsilon\|\nabla \xi\|_{L_{2}\left(0, t, L_{2}(\Omega)\right)}^{2}+ \\
+C\|\xi\|_{L_{2}\left(0, t, L_{2}(\Omega)\right)}^{2}
\end{gathered}
$$

The rest of the terms are estimated in the same way. Next, we take $\varepsilon=\frac{\gamma_{1}}{6}$. We substitute the obtained estimates in (14). Then, after reducing similar terms and taking into account the conditions of the theorem and inequalities (6) and (11), we derive the desired estimate (13). The theorem is proved.

\section{Conclusions}

When the condition of the ellipticity of the problem is satisfied, inequalities are proposed for the difference of the generalized solution of the considered parabolic problem with a divergent principal part and the solution of the auxiliary elliptic problem. Using these estimates and under additional conditions on the coefficients of problem (1), we obtain estimates of the error of the approximate solution of the Bubnov-Galerkin method in the norm $H^{1}(\Omega)$ of order $O\left(h^{k-1}\right)$ for the considered nonclassical parabolic problem with divergence the main part, when the boundary condition contains the time derivative of the desired function.

\section{References}

1. J. Kacur, Nonlinear parabolic equations with the mixed nonlinear and nonstationary boundary conditions, Math Slovoca 30, 213-237 (1980)

2. J. Kacur, Nonlinear parabolic boundary valve problems with the time derivative in the boundary conditions, Lect Notes Math 703, 170-178 (1979)

3. L.N. Tao, Heat conduction with nonlinear boundary condition, Z. angew. Math. Phys. 32, 144-155 (1981).

4. S.G. Mixlin, Numerical implementation of variational methods, Science, Moscow (1966)

5. J. Jr Douglas, T. Dupont, Galerkin methods for parabolic equations with nonlinear foundry conditions, NumerMalh. 20, 213-237 (1973)

6. J. E. Dench Jr, Galerking methods for some highly nonlinear problems, SIAM Numer Anal. 14, 327-434 (1977)

7. L. Jutchell, A Galerken method for nonlinear parabolic equations with nonlinear boundary conchtions, SIAM J Numer Anal. 16, 254-299 (1979)

8. I.M. Tikhinova, Application of the stationary galerkin method to the first boundary value problem for a mixed high-order equation, Mathematical notes of NEFU 23(4), 73-81(2016).

9. V.E. Fedorov, The stationary galerkin method applied to the first boundary value problem for a higher order equation with changing time direction, Mathematical Notes of NEFU 24(4), 67-75 (2017).

10. A.Z. Mamatov, Application of the Galerkin method to some quasilinear parabolic equation , Journal of LSU 13, 37-45 (1981) 
11. A.Z. Mamatov, G. Djumabaev, On a Problem of Parabolic Type with Divergent Principal Part, $53^{\text {rd }}$ International Scientific and Practical Conference, VGTU, Vitebsk, Belarus (2020)

12. A.Z. Mamatov, S. Baxramov, An approximate solution of the Galerkin method of a quasilinear equation with a boundary condition containing the time derivative of the required function, Uzbekistan -Malaysiya Joint Internatonal Scientific Conference, National University of Uzbekistan, Tashkent (2020).

13. A.Z. Mamatov, M.S. Dosanov, J. Raxmanov, D.X. Turdibaev, One parabolic problem with divergent principal part, National Association of Scientists 57, 59-63 (2020)

14. M.F. Wheeler, A priori error estimates for Galerkin approximation to parabolic partial differential equations, SIAM J. Numer. Anal. 10, 723-759 (1973)

15. V.G. Pimenov, A.B. Lojnikov, Numerical Methods, Publishing House of Yekaterinburg, Uralsk University, Uralsk, Russia (2014)

16. P.A. Denisov, V.F. Petrov, Numerical Methods: Study Guide, South-Russian State Polytechnic University named after M.I. Platova, Novocherkask (2017)

17. O.A. Ladyjenskaya, V.A. Solonnikov, N.N. Uraltseva, Linear and quasilinear equations of parabolic type, Science, Moscow (1967)

18. O.A. Ladyjenskaya, N.N. Uraltseva, Linear and quasilinear equations of elliptic type, Science, Moscow (1973)

19. A.D. Polyanin, W.E. Schiesser, A.I. Zhurov, Partial differential equations, Scholarpedia 3(10), 4605 (2008)

20. G. Burkhoff, M.H. Schultz, R.S. Varda, Piecewise Hermite interpolations in one and two variables with applications to partial differential equations, Numer Math 11, 232256 (1968)

21. J.H. Bramble, S.R. Hilbert, Bounds for a class of linear functionals with applications to Hermite interpolation, Numer Math 16, 362-369 (1971) 